\title{
Simulation Training in Minimally Invasive Direct Coronary Artery Bypass Grafting
}

\author{
Song Wu, MD, Yuan-hao Fu, MD, Hong Zhao, MD, Yun-peng Ling, MD \\ Cardiac Surgery Department, Peking University Third Hospital, Beijing, P.R. China
}

\section{ABSTRACT}

Background: To evaluate the effect of minimally invasive direct coronary artery bypass (MIDCAB) simulator for cardiac residency training.

Methods: A total of 26 resident surgeons who had never trained for coronary artery anastomosis participated in this training program. They received coronary artery anastomosis training on off-pump coronary artery bypass grafting (OPCAB) simulator for $15 \mathrm{~h}$. After training, their performance of anastomosis was evaluated on the OPCAB simulator according to 12 items and a 5-point global rating scale. Based on the total score of assessment, those with an individual score of 12-36 formed group A, while group B was composed of the remaining trainees. The two groups then received another $15 \mathrm{~h}$ coronary artery anastomosis training on the MIDCAB simulator, and the performance was assessed.

Results: Trainees improved their performance of coronary artery anastomosis after training on the OPCAB simulator. Group A was composed of 7 trainees with an individual with a total score of 12-36 points and group B was composed of the remaining 19 trainees. After MIDCAB simulator training, significant differences were noted in the pre- and posttraining values in the A group $(P<.001)$, and the assessment value of group A was significantly better than those of group B $(P<.05)$. No significant difference was detected between pre- and post-training values in group B after MIDCAB simulator training $(P>.05)$.

Conclusion: We concluded that trainees who performed well in OPCAB simulation training can also perform better in MIDCAB, and our designed MIDCAB simulator was useful for residency training.

\section{INTRODUCTION}

Coronary artery bypass grafting $(\mathrm{CABG})$ provides a longterm benefit to patients with advanced coronary artery disease. In China, OPCAB is widely performed because of its less invasiveness, especially in high-risk patients. However,

Received Fuly 12, 2020; received in revised form August 7, 2020; accepted August 7, 2020.

This study was supported by Incubation Project of Peking University Third Hospital Clinical Key Program (Y73536-02).

Correspondence: Yun-peng Ling, MD, Chief Surgeon, Cardiac Surgery Department, Peking University Third Hospital, 49 North Garden Rd, Haidian District, Beijing, P.R. China 100191; (e-mail: 13910193131@163.com). the conventional procedure is associated with potential complications and long duration of recovery. Minimally invasive direct coronary artery bypass grafting (MIDCAB) via small left thoracotomy has several advantages over CABG through median sternotomy in terms of early recovery, less bleeding, and no risk of sternal infection [Holzhey 2007; Kofidis 2009]. MIDCAB enables revascularization with a configuration similar to that of a sternotomy technique, using left internal thoracic artery (LITA) harvesting and hand-sewn proximal and distal anastomoses under direct visualization. Yet only a few institutes routinely perform MIDCAB due to technical challenges, which require the surgeons to perform an accurate and expeditious anastomosis on constantly moving target vessels in a narrow space. Also, cardiac surgeons need to be well-trained through this minimally invasive approach before attempting the procedure on patients. The use of animal models to gain proficiency in surgical skills is not costefficient or accepted due to legal and ethical concerns.
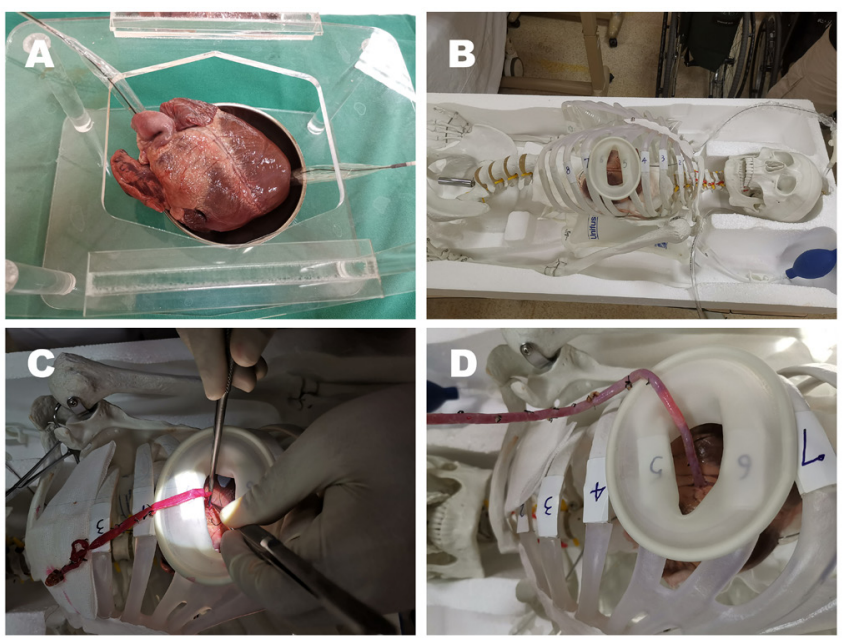

Figure 1. $A, O P C A B$ simulator. Isolated porcine heart was supported in our designed anastomosis training container. The balloon of the IABP catheter was inserted into the left ventricular cavity of the porcine heart from the aorta and passed through the apex. B, MIDCAB simulator. The disposable wound protector was placed in the midline of the left clavicle and 5 th intercostal incision of the skeleton. The isolated beating porcine heart was mounted in the thoracic cavity of the adult skeletal. C, MIDCAB was completed through wound protector the MIDCAB simulator. The saphenous vein was used to perform the coronary anastomosis with $L A D$ by the continuous running suture technique. $D$, On the MIDCAB simulator, the red ink was injected into the great saphenous vein to check the unobstructed anastomosis or the occurrence of anastomotic bleeding. 


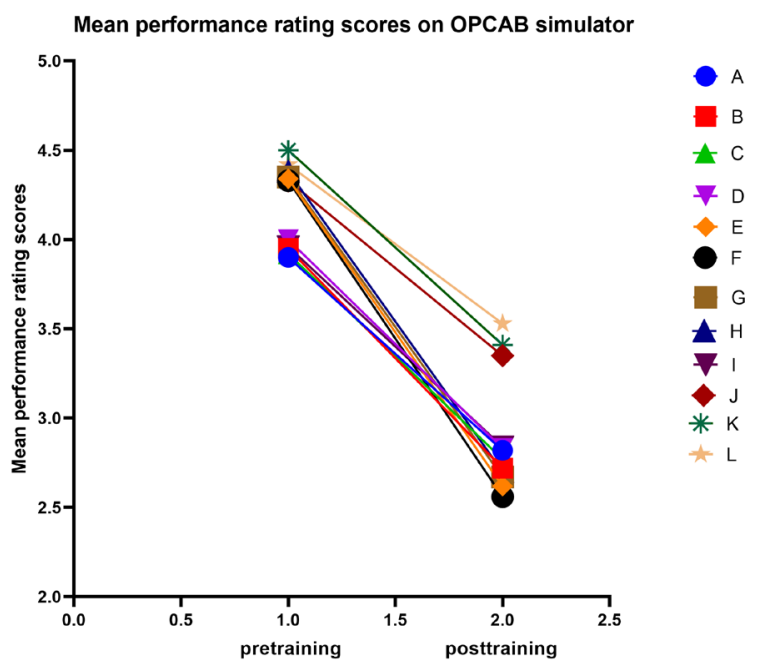

Figure 2. Spaghetti graph of mean performance-rating scores based on the assessment on the OPCAB simulator. The 26 trainees improved their performance of coronary artery anastomosis after training on the OPCAB simulator. Significant differences were noted in the assessment of pretraining and post-training values.

A: graft orientation, B: bite appropriate, C: spacing appropriate, D: coronary incision size, E: use of Castroviejo needle holder, F: use of forceps, G: needle angles, $\mathrm{H}$ : needle transfer, I: suture management/ tension, J: leakage of anastomosis, $\mathrm{K}$ : shape of anastomosis, L: completion time of anastomosis

Moreover, the thoracic structure of animals differs greatly from that of humans. Thus, simulation training devices may be a useful tool to provide the necessary practice for the surgeon outside the operating room [Murzi 2012]. Until now, there have been no simulation training devices suitable for MIDCAB [Fann 2010; Vaporciyan 2013; Nambiar 2018]. Based on our previously designed OPCAB simulator, we developed a MIDCAB training simulator and procedure. Thus, in this study, we evaluated the effect of our MIDCAB simulator for residency training and also tested the hypothesis that the OPCAB technique was the foundation of MIDCAB.

\section{MATERIALS AND METHODS}

This study was a blinded, prospective trial approved by our university. A total of 26 cardiac surgery residents and postgraduates from six hospitals affiliated with our university participated in this training program; none of them had received prior training in coronary artery anastomosis technology.

\section{Simulation Protocol}

The experiment includes two stages: OPCAB simulator training and MIDCAB simulator training. 26 cardiac surgery residents and postgraduates first received simulated training of coronary artery anastomosis on the OPCAB simulator for $15 \mathrm{~h}$ for 5 weeks. Subsequently, they were assessed on the OPCAB simulator by 12 items on a 5 -point global rating

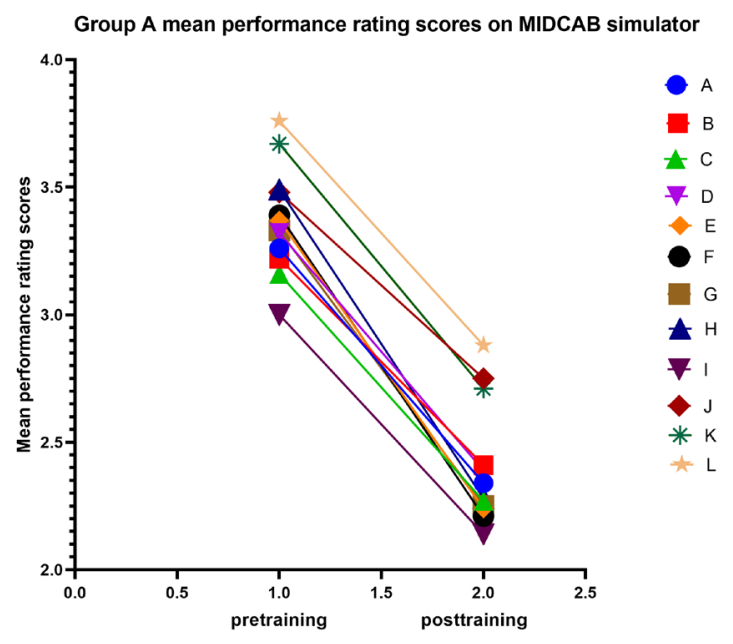

Figure 3. Spaghetti graph of mean performance-rating scores based on the
assessment on the MIDCAB simulator. Significant differences were noted
in the assessment of pretraining and post-training values in Group A.
A: graft orientation, B: bite appropriate, C: spacing appropriate, D: coro-
nary incision size, E: use of Castroviejo needle holder, F: use of forceps,
G: needle angles, H: needle transfer, I: suture management/tension, J:
leakage of anastomosis, K: shape of anastomosis, L: completion time of
anastomosis

scale. According to the total score of assessment, those with a total score of 12-36 constituted group A, and the remaining trainees formed group B. Both groups then underwent $15 \mathrm{~h}$ of simulated coronary artery anastomosis training on the MIDCAB simulator for 5 weeks, after which they were assessed on the MIDCAB simulator.

\section{OPCAB Simulator Training Stage}

The porcine hearts were obtained from abattoirs from animals slaughtered for consumption and prepared for usage. The remnants of the human saphenous vein were used as grafts for anastomoses. The remnants of vein specimens were acquired after obtaining consent for research. Porcine hearts were prepared and supported in our designed anastomosis training container (Chinese National Utility Model patent 2014206891414). In order to simulate the clinical scene of OPCAB, the catheter of the balloon of intraaortic balloon pump (IABP) was inserted into the left ventricular cavity of the porcine heart from the aorta and passed through the apex (Figure 1, A). The other end of the IABP catheter was connected to the IABP machine. The balloon was connected to a console that regulated the inflation or deflation of the balloon with the passage of helium. The volume of the balloon was $40 \mathrm{~mL}$, which was controlled by regulating the volume of inflation. Then the amplitude of the porcine heart beating was adjusted according to the left ventricular cavity size and operational requirements. The built-in frequency of the IABP machine ( 80 beats/min) was used. In this experiment, the left anterior descending artery (LAD) was used as the target vessel for training and assessment. The location of 


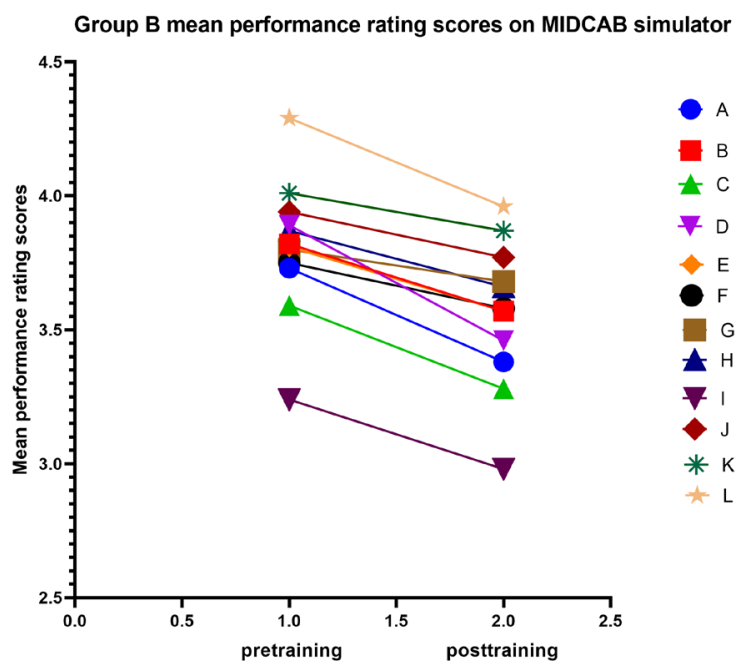

Figure 4. Spaghetti graph of mean performance-rating scores based on the assessment on MIDCAB simulator. Although trainees have improved their performance of coronary artery anastomosis after training, no significant difference was detected between pretraining and post-training values in Group B.

A: graft orientation, B: bite appropriate, C: spacing appropriate, D: coronary incision size, E: use of Castroviejo needle holder, $\mathrm{F}$ : use of forceps, G: needle angles, $\mathrm{H}$ : needle transfer, I: suture management/ tension, J: leakage of anastomosis, K: shape of anastomosis, L: completion time of anastomosis

LAD was relatively constant, and the diameter of the vessel was large, which was beneficial for beginners to learn and master. The trainees were educated about the technique for exposing the LAD and performing arteriotomy and distal end-to-side anastomosis. A shunt was routinely used after coronary arteriotomy on the beating porcine heart. The distal end of the saphenous vein segment was beveled, and a continuous running suture technique was used to perform the coronary anastomosis. Subsequently, the role of the operator and the assistant was reversed.

\section{MIDCAB Simulator Training Stage}

In order to simulate the clinical scene of MIDCAB, the isolated porcine heart was mounted in the thoracic cavity of the adult skeletal specimen (height $180 \mathrm{~cm}$ ), where the normal heart was located. The balloon of the IABP catheter was inserted into the porcine heart similar to that of the OPCAB simulator. The apex of the porcine heart was at the 5 th intercostal level of the middle clavicle, and the distance between the surface of the porcine left ventricle and the skeletal specimen was $5-6 \mathrm{~cm}$. The adult skeletal specimen was placed in a supine position and rotated $30^{\circ}$ decubitus towards the right side (Figure 1, B). The disposable wound protector was placed in the midline of the left clavicle and the 5th intercostal incision of the skeleton. MIDCAB was completed through simulating a small, left anterolateral thoracotomy [Nambiar 2018; Lou 2013; Lou 2014] (Figure 1, C).
Table 1. Performance Rating Scores

Performance-Rating

Scores

(A) Graft orientation (proper orientation for toeheel, appropriate start and end points)

(B) Bite appropriate (entry and exit points, number of punctures, even and consistent distance from edge)

(C) Spacing appropriate (uniform spacing, consistent distance from previous bite, too close versus too far)

(D) Coronary incision size (compared to graft) and orientation

(E) Use of Castroviejo needle holder (finger placement, instrument rotation facility, needle placement pronation and supination, proper finger and hand motion, lack of wrist motion)

(F) Use of forceps (facility, hand motion, assist needle placement, appropriate traction on tissue)

(G) Needle angles (proper angle relative to tissue $\begin{array}{llllll}\text { and needle holder, consider the depth of field, antici- } & 1 & 2 & 3 & 4 & 5\end{array}$ pating subsequent angles)

$(\mathrm{H})$ Needle transfer (needle placement and preparation from stitch to stitch, use of instrument and hand $\begin{array}{llllll}1 & 2 & 3 & 4 & 5\end{array}$ to mount needle)

(I) Suture management/tension (too loose versus too tight, use tension to assist exposure, avoid entanglement)

(J) Leakage of anastomosis(1, none; 2, oozing; 3, 1-point leak; 4, 2-point leak; 5, dehiscence)

(K) Shape of anastomosis (1, cobra head; 2, flat; 3 , deformation; 4, stenosis; 5 , obstruction)

(L) Completion time of anastomosis (1, $<5 \mathrm{~min} ; 2$, 6-10 min; 3, 11-15 min; 4, 16-19 min; 5, >20 min)

Scores: 1, excellent, able to accomplish goal without hesitation, showing excellent progress and flow; 2, good, able to accomplish goal deliberately, with minimal hesitation, showing good progress and flow; 3 , average, able to accomplish goal with hesitation, discontinuous progress and flow; 4 , below average, able to partially accomplish goal with hesitation; 5, poor, unable to accomplish goal, marked hesitation.

\section{Performance Assessment}

The instructors were responsible for recording the time of anastomosis taken by each trainee and the final quality of the blood vessels. In order to reduce the bias of the experimental results, we stipulated the training time and intensity of each trainee to maintain consistency. The performance of anastomosis was evaluated according to a 5 -point global rating scale (1: excellent, 2: good, 3: average, 4: below average, 5: poor), and assessment indicators included 12 items, with a total score of 60 points (Table 1). The attending surgeons were instructed about the use of the 5-point rating scale, which was modified from the objective structured 
Table 2. Mean Performance-Rating Scores According to Subsequent Review of the Video Recordings on OPCAB Imulator

\begin{tabular}{|c|c|c|c|c|}
\hline (1) Graft orientation & 0.76 & $3.90 \pm 0.92$ & $2.82 \pm 0.42$ & .015 \\
\hline (3) Spacing appropriate & 0.70 & $3.92 \pm 1.13$ & $2.71 \pm 0.38$ & .001 \\
\hline (4) Coronary incision size & 0.72 & $4.00 \pm 0.70$ & $2.83 \pm 0.32$ & .010 \\
\hline (6) Use of forceps & 0.65 & $4.33 \pm 0.60$ & $2.56 \pm 0.48$ & .000 \\
\hline (7) Needle angles & 0.65 & $4.35 \pm 0.57$ & $2.67 \pm 0.35$ & .000 \\
\hline (8) Needle transfer & 0.71 & $4.38 \pm 0.50$ & $2.70 \pm 0.51$ & .000 \\
\hline (9) Suture management/tension & 0.80 & $3.96 \pm 1.10$ & $2.84 \pm 0.55$ & .007 \\
\hline (10) Leakage of anastomosis & 0.73 & $4.33 \pm 0.83$ & $3.35 \pm 1.23$ & .031 \\
\hline
\end{tabular}

Data are expressed as mean $\pm S D$. Paired $t$ test was performed for baseline versus end. For all comparisons, $P<.001$.

assessment of technical skills (OSATS). The components of this rating scale included graft orientation, bites, spacing, use of needle holder, use of forceps, needle angles, needle transfer, suture management, and tension. The red ink was injected into the great saphenous vein to check the unobstructed anastomosis or the occurrence of anastomotic bleeding (Figure 1, D). Finally, in the proximity of anastomosis, the great saphenous vein was cut off. Then, the anastomosis was detected by the coronary artery probe from the cavity and deformation, narrowing, distortion, and occlusion. The performance of trainees was recorded with a digital video camera and stored for subsequent review. The video data were rated according to the 5-point global rating scale by three experienced surgeons in a blinded manner. The interobserver reliability of reviewers was evaluated using Cohen's kappa statistics on OPCAB and MIDCAB simulator [Liu 2014; Baker 2012; Nesbitt 2013; Cavalli 2011; Sadeghifar 2018; Fann 2012].

\section{Statistical Analysis}

We evaluated whether the data were normal distribution. For continuous variables, Student $t$ test and paired $t$ test were used to estimate the differences in the normal distribution. The data were presented as mean $\pm \mathrm{SD}$, and paired $t$ test was performed to assess pretraining versus posttraining values; $\mathrm{P}<.05$ indicated statistical significance. The interobserver reliability of reviewers was evaluated using Cohen's kappa statistics on OPCAB and MIDCAB training simulator. The results were analyzed using SPSS version 24 software (IBM, Armonk, NY, USA) by reliability analysis tests and kappa measurement. Weighted kappa statistics were defined as follows [Cavalli 2011; Sadeghifar 2018]: $\leq 0$ indicates poor reliability; 0.01-0.2, slight; $0.21-$ 0.4 , fair; $0.41-0.6$ moderate; $0.61-0.8$, substantial; $0.81-1$, almost perfect.

\section{RESULTS}

According to the $\mathrm{OPCAB}$ training simulator, kappa values ranged from 0.64 through 0.80 between reviewers, which revealed good interobserver reliability. Experimental results showed that trainees improved their performance of coronary artery anastomosis after training on the OPCAB simulator. Significant differences were noted in the assessment of pretraining and post-training values $(P<.001$ for all comparisons) (Table 2; Figure 2).

After receiving $\mathrm{OPCAB}$ simulator training, the trainees were divided into two groups according to the OPCAB test results. Group A comprised 7 trainees, each with a total score of 12-36 points in the OPCAB test and Group B consisted of the remaining 19 trainees. Then, the two groups entered the MIDCAB simulator training phase. On the training simulator, the kappa values ranged from 0.61 through 0.74 between the reviewers, which also revealed satisfactory interobserver reliability. Experimental results showed that trainees improved their performance of coronary artery anastomosis after training on MIDCAB simulator. Significant differences were noted in the assessment of pretraining and post-training values in the $\mathrm{A}$ group $(P<.001$ for all comparisons) (Figure $3)$. Interestingly, the assessment value of Group A was significantly better than that of Group B $(P<.05)$. Although Group $B$ showed that trainees had improved the performance of coronary artery anastomosis after MIDCAB simulator training, no significant difference was detected between pre and posttraining values (Table 3 and Figure 4).

\section{DISCUSSION}

Although CABG has been shown to have better survival, and repeat revascularization, many patients still prefer 
Table 3. Mean Performance-Rating Scores According to Subsequent Review of the Video Recordings on MIDCAB Simulator

\begin{tabular}{|c|c|c|c|c|c|c|c|c|}
\hline & & $A(r$ & $=7)$ & $B(n$ & $=19)$ & & $P$ & \\
\hline (1) Graft orientation & 0.69 & $3.26 \pm 0.47$ & $2.34 \pm 1.0$ & $3.73 \pm 0.58$ & $3.38 \pm 0.79$ & .0201 & .7016 & .0091 \\
\hline (2) Bite appropriate & 0.68 & $3.22 \pm 0.45$ & $2.41 \pm 0.56$ & $3.82 \pm 0.52$ & $3.57 \pm 0.63$ & .0287 & .8254 & .0012 \\
\hline (4) Coronary incision size & 0.63 & $3.32 \pm 0.58$ & $2.39 \pm 0.47$ & $3.89 \pm 0.42$ & $3.46 \pm 0.94$ & .0194 & .3144 & .0015 \\
\hline (5) Use of Castroviejo needle holder & 0.70 & $3.37 \pm 0.43$ & $2.24 \pm 0.72$ & $3.81 \pm 0.62$ & $3.57 \pm 0.58$ & .0001 & .3569 & .0010 \\
\hline (6) Use of forceps & 0.73 & $3.39 \pm 0.88$ & $2.21 \pm 0.67$ & $3.75 \pm 0.47$ & $3.58 \pm 0.66$ & .0001 & .5380 & .0001 \\
\hline (7) Needle angles & 0.68 & $3.33 \pm 0.48$ & $2.25 \pm 0.74$ & $3.80 \pm 0.68$ & $3.68 \pm 0.73$ & .0001 & .6101 & .0010 \\
\hline (11) Shape of anastomosis & 0.61 & $3.67 \pm 0.75$ & $2.71 \pm 0.77$ & $4.01 \pm 0.74$ & $3.87 \pm 0.96$ & .0373 & .8230 & .0215 \\
\hline (12) Completion time of anastomosis & 0.68 & $3.76 \pm 0.63$ & $2.88 \pm 0.63$ & $4.29 \pm 0.52$ & $3.96 \pm 1.21$ & .0394 & .6874 & .0110 \\
\hline
\end{tabular}

Data are expressed as mean \pm SD. Paired $t$ test was performed for pretraining data versus post-training data. The parameters were compared between the two groups in the pretraining and post-training. *For comparisons, $\mathrm{P}<.05$.

percutaneous coronary intervention (PCI) due to faster improvement of the health status and ability to return to normal daily activity after PCI. Therefore, a less invasive technique that provides the protective effect of CABG while avoiding sternotomy is an urgent requirement. MIDCAB is one of the novel CABG approaches that enable a minimally invasive approach to coronary revascularization through a left small thoracotomy. The short and midterm clinical outcomes and graft patency are excellent with a short length of hospital stay, less infection of the surgery site, and rapid postoperative recovery [Repossini 2019; Raja 2018; Babliak 2020]. The satisfactory results encouraged us to adopt the technique of MIDCAB in 2012. From February 2012 to November 2019, a total of 620 CABG cases underwent MIDCAB procedures in our center [Zhang 2016; Wu 2017].

Despite the safety and efficacy, MIDCAB did not become a standard procedure for several reasons, mainly due to the technically demanding nature. It is a combination of OPCAB technique and minimally invasive procedure, which requires the operators to perform an accurate and expeditious anastomosis on constantly moving target vessels in a narrow tunnel space. The cardiac surgeon must be well-trained to attempt this MIDCAB technique on patients. The operating room might not be an ideal location for early surgical training due to ethical concerns, time constraints, and complex procedures performed on high-risk patients [Lou 2013]. Thus, simulation-based learning might provide a surgeon with necessary training and practice outside the operating room, in order to gradually adapt to the narrow tunnel space and field of vision for coronary artery anastomosis on the beating heart.
Two categories of surgical simulators for CABG training include the simple bench model (SBM), and human performance simulator (HPS). SBMs are "partial-task" tools that simulate a small component of a larger operation. They may be synthetic or consist of biological tissue. Use of biological tissues in many of these models decreases cost- and timeefficiency and may not accurately mimic human anatomy [Reznick 2006]. Early beating-heart SBMs, such as the Zurich Heart-Trainer and Izzat off-pump CABG model, paved the way for the development in beating heart models [Reuthebuch 2002; Izzat 1998]. One such model is the Chamberlain beating heart trainer described by The Chamberlain Group. This sutureable rubber model has an anatomically realistic appearance and is able to contract at different speeds and rhythms to serve as a high-fidelity SBM [Fann 2010; Fann 2012]. The advantages of SBMs include a simple physical model for basic tasks and inexpensive, and disadvantages include incomplete surgical experience.

The HPS is a high-technology system that fuses an elaborate physical component with a computer interface. These systems typically simulate the entire OR environment and are used for both individual and team training of an operation from start to finish [Reznick 2006]. HPS can include patient variation and capabilities for assessment and feedback. The Ramphal Cardiac Surgery Simulator is a perfused, beating heart simulator used most extensively for CABG. It includes a porcine heart with the right and left ventricles filled with balloons that are connected to a computer-controlled pneumatic pump, allowing their inflation to simulate pulsation of the heart. Artificial blood perfuses the entire system, including 
the coronary arteries and veins, atria, and ventricles via a perfusion line connected to a roller pump. The advantages of HPS include clinical variation and user assessment, capacity for team training; the disadvantages of HPS may involve expensive expenses, extensive setup, and downtime.

MIDCAB has accounted for more than $50 \%$ of all CABG operations in our hospital, so we often faced the problem of carrying out the MIDCAB training program for our cardiac surgeons. Our limited experience told us that the surgeon should first be an expert in median sternotomy OPCAB surgery before attempting $M I D C A B$ procedure. Our MIDCAB training program include $\mathrm{OPCAB}$ simulator and $\mathrm{MIDCAB}$ simulator training stage. The OPCAB simulator mainly consists of isolated porcine heart, the catheter of the balloon, and IABP machine. The amplitude and frequency of the porcine heart beating was adjusted according to the operational requirements. The MIDCAB simulator was constructed by placing the OPCAB simulator in the chest of an adult skeleton. The trainees could perform coronary artery anastomosis under direct vision through a wound protector placed in the midline of left clavicle and 5 th intercostal of the skeleton.

In this study, we tested the hypothesis that the OPCAB technique was the foundation of MIDCAB procedure, and our designed MIDCAB simulator was useful for residency training. Before starting the MIDCAB training, we first organized the trainees to carry out the $\mathrm{OPCAB}$ training on the OPCAB simulator. The beginners learned how to expose and incise the moving target coronary artery, expand the incision, and place the intracoronary artery shunt. They also practiced how to use Castroviejo microscissors, Castroviejo microneedle holder, and microforceps to perform anastomosis. The study showed significant differences in the assessment of preand post-training values of the application of instruments, anastomotic speed, and anastomotic quality. We speculated that the training on the OPCAB simulator might be helpful for trainees to improve the coordination of needle holder and forceps, as well as the coordination of eyes and hands, especially in the selection of needle insertion site, the angle of needle insertion, as well as how forceps assist in needle extraction on the moving target vessel.

The trainees were divided into two groups according to the OPCAB test results, and then both groups entered the MIDCAB simulator training phase. Significant differences were noted in the baseline and post-training values after MIDCAB simulator training in trainees who performed well in OPCAB simulation training. And they were also superior to those who performed worse in OPCAB simulation training with respect to the application of microsurgical instruments, anastomotic speed, and distal anastomotic quality after MIDCAB simulator training stage. The skilled application of microsurgical needle holder and forceps would help trainees to control the stitches freely, which would guarantee the shape of distal anastomosis with standard "cobra-head" technique at an oblique angle to the moving targets through the narrow tunnel space. Theoretical advantages of such an anastomosis included improvement of flow characteristics and decreased tendency to thrombosis formation, which was directly related to the long-term patency of coronary grafts
[Matsuura 2019; Ghista 2013]. The technique of MIDCAB was associated with a learning curve regarding the operative time; the quality of anastomotic vessels could be improved, as well as the operative time shortened by MIDCAB simulator training. It can be speculated that trainees who performed well in OPCAB simulation training can also perform better in MIDCAB, which was consistent with the actual situation in the clinic. Whether training on beating heart condition could help trainees to overcome the involuntary handshaking and emotional tension was not supported by direct measurement results.

In practice, we found that our designed MIDCAB simulator was a good training platform that did not need complex equipment and specialized laboratories, and the young cardiac surgeon could be trained in spare time, which can be taken home or in the office as homework. Skills and tasks taught in using this surgical training simulator can translate to the clinical setting. The trainees reported confidence in their ability to perform MIDCAB after using our designed simulator. However, simulation is not meant to eliminate the need for genuine patient interaction and real OR experience. Acknowledging the limitations of simulation-based training, the simulator can never mimic the feel of live human tissue and the complexity of human physiology. But it might serve as a crucial adjunct or bridge for the advanced technique, with a safer transition to daily clinical practice [Enter 2015; Mokadam 2017; Feins 2017; Ribeiro 2017; Ramphal 2005]. The simulation does not mimic the feel of living human tissue and the complexity of human physiology. This MIDCAB simulator only simulates the ideal conditions in the process of operation but does not stimulate some operative events, such as bleeding, obscured view, and hemodynamic fluctuation. Therefore, this study aimed to provide a simulation training tool and a strategy for the surgical teams interested in developing the MIDCAB technique.

\section{Conclusion}

We conclud that trainees who performed well in OPCAB simulation training can also perform better in MIDCAB, and our designed MIDCAB simulator was useful for the training of cardiac surgeons training, providing satisfactory simulation results.

\section{ACKNOWLEDGEMENTS}

We thank Hang Yang, MD, and Yi-chen Gong, MD, for their support and help in teaching experiments, data collection, and statistical processing.

\section{REFERENCES}

Babliak O, Demianenko V, Melnyk Y, et al. 2020. Multivessel arterial revascularization via left anterior thoracotomy. Semin Thorac Cardiovasc Surg 27 pii: S1043-0679(20)30047-2.

Baker CJ, Sinha R, Sullivan ME. 2012. Development of a cardiac surgery simulation curriculum: from needs assessment results to practical implementation. J Thorac Cardiovasc Surg 144:7-16. 
Cavalli F, Izadi A, Ferreira AP, et al. 2011. Interobserver Reliability among Radiologists and Orthopaedists in Evaluation of Chondral Lesions of the Knee by MRI. Adv Orthop 2011:743742.

Enter DH, Lou X, Hui DS, et al. 2015. Practice improves performance on a coronary anastomosis simulator, attending surgeon supervision does not. J Thorac Cardiovasc Surg 149:12-6, 17.e1-2.

Fann JI, Calhoon JH, Carpenter AJ, et al. 2010. Simulation in coronary artery anastomosis early in cardiothoracic surgical residency training: the boot camp experience. J Thorac Cardiovasc Surg 139:1275-81.

Fann JI, Feins RH, Hicks GL Jr, et al. 2012. Senior tour in cardiothoracic surgery. Evaluation of simulation training in cardiothoracic surgery: the senior tour perspective. J Thorac Cardiovasc Surg 143:264-72.

Feins RH, Burkhart HM, Conte JV, et al. 2017. Simulation-based training in cardiac surgery. Ann Thorac Surg 103:312-21.

Ghista DN, Kabinejadian F. 2013. Coronary artery bypass grafting hemodynamics and anastomosis design: a biomediacal engineering review. Biomed Eng Online 13:129.

Holzhey DM, Jacobs S, Mochalski M, et al. 2007. Seven-year follow-up after minimally invasive direct coronary artery bypass: experience with more than 1300 patients. Ann Thorac Surg. 83:108-14.

Izzat MB, EI-Zufari MH, Yim AP. 1998. Training model for beating heart coronary artery anastomoses. Ann Thorac Surg 66:580-1.

Kofidis T, Emmert MY, Paeschke HG, et al. 2009. Long-term follow-up after minimal invasive direct coronary artery bypass grafting procedure: a multi-factorial retrospective analysis at 1000 patient-years. Interact Cardiovasc Thorac Surg 9:990-4.

Liu X, Yang Y, Meng Q, et al. 2016. Secure and high-fidelity live animal model for off-pump coronary bypass surgery training. J Surg Educ 73:583-8.

Lou X, Enter D, Sheen L, et al. 2013. Sustained supervised practice on a coronary anastomosis simulator increases medical student interest in surgery, unsupervised practice does not. Ann Thorac Surg 95:2057-63.

Lou X, Lee R, Feins RH, et al. 2014. Training less-experienced faculty improves reliability of skills assessment in cardiac surgery. J Thorac Cardiovasc Surg 148:2491-6.e1-2.

Matsuura K, Jin WW, Liu H, et al. 2019. Computational fluid dynamic study of different incision length of coronary artery bypass grafting in a native coronary stenosis model. J Thorac Dis 11:393-9.

Mokadam NA, Fann JI, Hicks GL, et al. 2017. Experience with the cardiac surgery simulation curriculum: results of the resident and faculty survey. Ann Thorac Surg 103:322-8.
Murzi M, Caputo M, Aresu G, et al. 2012. Training residents in off-pump coronary artery bypass surgery: a 14-year experience. J Thorac Cardiovasc Surg 143:1247-53.

Nambiar P, Kumar S, Mittal CM, et al. 2018. Minimally invasive coronary artery bypass grafting with bilateral internal thoracic arteries: Will this be the future? J Thorac Cardiovasc Surg 155:190-7.

Nesbitt JC, St Julien J, Absi TS, et al. 2013. Tissue-based coronary surgery simulation: medical student deliberate practice can achieve equivalency to senior surgery residents. J Thorac Cardiovasc Surg 145:1453-8; discussion 1458-9.

Raja SG, Garg S, Rochon M, et al. 2018. Short-term clinical outcomes and long-term survival of minimally invasive direct coronary artery bypass grafting. Ann Cardiothorac Surg 7:621-7.

Ramphal PS, Coore DN, Craven MP, et al. 2005. A high fidelity tissuebased cardiac surgical simulator. Eur J Cardiothorac Surg 27:910-6.

Repossini A, Di Bacco L, Nicoli F, et al. 2019. Minimally invasive coronary artery bypass: twenty-year experience. J Thorac Cardiovasc Surg 158:127-38.

Reuthebuch O, Lang A, Groscurth P, et al. 2002. Advanced training model for beating heart coronary artery surgery: the Zurich heart-trainer. Eur J Cardiothorac Surg 22:2448-8.

Reznick RK, MacRae H. 2006. Teaching surgical skills-changes in the wind. N Engl J Med 355:2664-9.

Ribeiro IB, Ngu JMC, Gill G, et al. 2017. Development of a high fidelity pressurized porcine beating heart simulator for cardiac surgery training. Perfusion 32:568-73.

Sadeghifar A, Kahani AK, Saied A, et al. 2018. Interobserver and intraobserver reliability of different methods of examination for presence of palmaris longus and examination of fifth superficial flexor function. Anat Cell Biol 51:79-84.

Vaporciyan AA, Yang SC, Baker CJ, et al. 2013. Cardiothoracic surgery residency training: past, present, and future. J Thorac Cardiovasc Surg 146:759-67.

Wu S, Ling Y, Fu Y, et al. 2017. Mid-term follow-up outcomes of 2-staged hybrid coronary revascularization compared with off-pump coronary artery bypass for patients with multivessel coronary artery disease. Wideochir Inne Tech Maloinwazyjne 12:178-185.

Zhang L, Cui Z, Song Z, et al. 2016. Minimally invasive direct coronary artery bypass for left anterior descending artery revascularization -analysis of 300 cases. Wideochir Inne Tech Maloinwazyjne 10: 548-54. 